\title{
Orquiectomía en pacientes pediátricos. Reporte de 12 años en un hospital de tercer nivel de la Ciudad de México
}

\section{Orchiectomy in pediatric patients. Report on 12 years of experience at a tertiary care hospital in Mexico City}

\author{
Alfonso Fernando Galván-Montaño, ${ }^{1}$ María de Lourdes Suárez-Roa, ${ }^{2}$ Marco Antonio Vela-Vázquez, ${ }^{3}$ Marco \\ Antonio Blancas-Rivera, ${ }^{3}$ Silvia García-Moreno ${ }^{2}$
}

\begin{abstract}
Resumen
ANTECEDENTES: Las alteraciones testiculares en pacientes pediátricos son trastornos frecuentes. Estas enfermedades se dividen en congénitas (testículo evanescente y atrofia testicular) y adquiridas (torsión testicular y tumores).

OBJETIVO: Determinar las causas relacionadas con la práctica de la orquiectomía en pacientes pediátricos.

MATERIALES Y MÉTODOS: Estudio descriptivo, retrospectivo y transversal, Ilevado a cabo a partir de la revisión de expedientes de pacientes pediátricos operados para orquiectomía en el Hospital General Dr. Manuel Gea González, entre los años 2002 a 2014. Se evaluaron las variables de edad, localización del testículo afectado, reporte de estudios de imagen (ultrasonido o tomografía), complicaciones quirúrgicas, diagnóstico histológico y colocación de prótesis testiculares. En pacientes con diagnóstico de torsión testicular se obtuvo la información del tiempo transcurrido entre el inicio de los síntomas hasta el momento de la intervención quirúrgica y en los pacientes con tumor testicular el antecedente de criptorquidia y tipo de procedimiento quirúrgico efectuado en el testículo evanescente. Para el análisis estadístico se estimaron solamente los porcentajes.

RESULTADOS: Se registraron 60 expedientes: 24 (40\%) con diagnóstico de torsión testicular, $14(23 \%)$ con atrofia testicular, $12(20 \%)$ con tumor testicular y $3(5 \%)$ con testículo evanescente; además, se encontró $1(1 \%)$ caso con resto mülleriano y 6 con testículos normales ( 5 con diagnóstico preoperatorio de atrofia testicular y 1 con torsión testicular).
\end{abstract}

CONCLUSIONES: El porcentaje de pacientes con alteraciones testiculares que requieren orquiectomía es elevado (27\%); su principal causa se debe a torsión testicular.

PALABRAS CLAVE: Pacientes pediátricos; orquiectomía; alteraciones testiculares.

\section{Abstract}

BACKGROUND: Testicular pathologies in children are frequent and can be catalogued as congenital and acquired. The congenital defects that require orchiectomy are vanishing testis and testicular atrophy, and the acquired ones are testicular torsion and testicular tumors.

OBJECTIVE: The aim of the present study was to determine the indications for orchiectomy performed on pediatric patients.

MATERIALS AND METHODS: The case records of pediatric patients that underwent orchiectomy at the Hospital General Manuel Gea González within the time frame of 2002 to 2017 were reviewed in a descriptive, retrospective, cross-sectional study. The variables of age, location of the affected testis, imaging study reports (ultrasound and tomography), surgical complications, histologic diagnosis, and testicular prosthesis

\footnotetext{
${ }^{1}$ Departamento de Cirugía pediátrica. 2 Departamento de Investigación clínica. ${ }^{3}$ Departamento de Pediatría.
}

Hospital General Dr. Manuel Gea González, Universidad Nacional Autónoma de México (UNAM), Ciudad de México.

Recibido: noviembre 2017

Aceptado: mayo 2018

Correspondencia

Alfonso Fernando Galván Montaño gamagq3@hotmail.com

Este artículo debe citarse como Galván-Montaño AF, Suárez-Roa ML, Vela-Vázquez MA, Blancas-Rivera MA, García-Moreno S. Orquiectomía en pacientes pediátricos. Reporte de 12 años en un hospital de tercer nivel de la Ciudad de México. Rev Mex Urol. 2018 mayo-junio;78(3):183-188. DOI:https://doi.org/10.24245/revmexurol.v78i3.1794 
placement were evaluated. The information on patients diagnosed with testicular torsion included the time interval from symptom onset to surgical intervention. In patients with testicular tumor, a history of cryptorchidism was determined and in cases of vanishing testis, the surgical approach was registered. Only percentages were calculated for the statistical analysis.

RESULTS: Sixty case records were reviewed. There were $24(40 \%)$ patients diagnosed with testicular torsion, 14 (23\%) with testicular atrophy, $12(20 \%)$ with testicular tumor, and $3(5 \%)$ with vanishing testis. One (1\%) case presented with Müllerian remnants and there were 6 cases of normal testes ( 5 preoperatively diagnosed with testicular atrophy and one with testicular torsion).

CONCLUSIONS: The percentage of testicular pathology requiring orchiectomy at our hospital was high $(27 \%)$ and its main cause was testicular torsion.

KEYWORDS: Pediatric patients; Orchiectomy; Testicular pathologies.

\section{ANTECEDENTES}

Las alteraciones testiculares en pacientes pediátricos son trastornos frecuentes, incluso pueden requerir tratamiento quirúrgico (orquiectomía). Este tipo de enfermedades se dividen en congénitas y adquiridas. ${ }^{1-3}$ Entre las congénitas que requieren orquiectomía se encuentran el testículo evanescente y la atrofia testicular. La primera se aplica a pacientes con testículos que tuvieron pérdida del aporte sanguíneo y generalmente se debe a torsión testicular perinatal. ${ }^{4,5}$ La atrofia testicular significa una disminución total o parcial del número de células o tamaño del órgano y puede ocurrir durante el periodo prenatal o posnatal, ya sea por una torsión testicular o posterior a orquidopexia efectuada para corregir un caso de criptorquidia o, bien, en pacientes que requieren tratamiento quirúrgico con técnica Fowler-Stephens para testículos intraabdominales, en quienes la posibilidad de atrofia puede ser de $35 \% .^{6}$

Por su parte, las alteraciones adquiridas incluyen la torsión testicular y los tumores. La primera ocurre ante un evento de rotación del cordón espermático, que obstruye el retorno venoso y posteriormente flujo arterial, lo que resulta en isquemia testicular. La incidencia de esta altera- ción es de 1 por cada 4000 hombres menores de 25 años y puede aparecer desde el nacimiento hasta los 15 años de edad (en promedio a los 9 años). ${ }^{7}$ El traumatismo testicular directo es un factor relacionado con la torsión testicular en 4\% de los casos. ${ }^{8,9}$ El testículo izquierdo se afecta en $70 \%$ de los pacientes y aunque la torsión unilateral es más frecuente, pueden aparecer en casos esporádicos con torsión bilateral. La isquemia se inicia en las primeras 4 horas; $\sin$ embargo, 90\% de los testículos pueden salvarse mediante intervención quirúrgica en las primeras 6 horas del evento, 50\% en las primeras 12 horas y menos de $10 \%$ después de las 24 horas; aproximadamente $87 \%$ de los casos requiere orquiectomía. $^{10,11}$

En cuanto a los tumores malignos durante la edad pediátrica, las alteraciones testiculares corresponden a $20 \%$ y las leucemias a $30 \%$ de las neoplasias, principalmente la leucemia linfoblástica, que suele afectar a niños de 9 años de edad (límites de 5 a 20 años). ${ }^{12}$ La segunda neoplasia concierne al rabdomiosarcoma (17\%), una tumoración sólida frecuente en pacientes de 11 años de edad. ${ }^{12}$ Los tumores de células germinales son la tercera tumoración más común en adolescentes de 16.6 años de edad (límites de 15 a 19 años). ${ }^{12}$ Para el grupo de 6 meses de 
edad, la neoplasia sólida con mayor incidencia corresponde al tumor de células granulosas, con un límite de 3 días a 5 meses de edad; después de los 6 meses, los tumores más frecuentes suponen el teratoma y el tumor del saco vitelino. ${ }^{12}$ Este último también se conoce como tumor del saco endodérmico, carcinoma juvenil embrionario o mesoblastoma extraembrionario. Entre las alteraciones menos frecuentes se encuentran el neuroblastoma, hamartoma y seminoma. ${ }^{12}$

Para los patólogos, urólogos y cirujanos pediatras en general, es importante conocer la repercusión de las lesiones testiculares y la edad en que se manifiestan, pues para cada alteración existe una descripción médica detallada; sin embargo, hay pocas publicaciones relacionadas con estas enfermedades y su prevalencia relativa. Hasta el momento se han efectuado tres estudios que aportan la información en este campo, principalmente en Estados Unidos, Australia e Inglaterra. ${ }^{1,12,13}$ No tenemos conocimiento de que exista en México información al respecto, por lo que es importante analizar la prevalencia y las causas de la orquiectomía en pacientes pediátricos, con la finalidad de aportar conocimiento para la toma de decisiones.

Por lo anterior, el objetivo de este estudio fue: determinar las causas relacionadas con la práctica de la orquiectomía en pacientes pediátricos que acuden al Hospital General Dr. Manuel Gea González.

\section{MATERIALES Y MÉTODOS}

Estudio descriptivo, retrospectivo y transversal, llevado a cabo a partir de la revisión de los expedientes de pacientes pediátricos a quienes se practicó orquiectomía en el Hospital General Dr. Manuel Gea González, entre los años 2002 a 2014. De la información recabada se evaluaron las variables de edad, localización del testículo afectado, reporte de estudios de imagen (ultrasonido o tomografía), complicaciones quirúrgicas, diagnóstico histológico y colocación de prótesis testiculares. En pacientes con diagnóstico de torsión testicular se obtuvo la información del tiempo transcurrido entre el inicio de los síntomas hasta el momento de la intervención quirúrgica y en los pacientes con tumor testicular el antecedente de criptorquidia y tipo de procedimiento quirúrgico efectuado en el testículo evanescente. Para el análisis estadístico se estimaron solamente los porcentajes.

\section{RESULTADOS}

Se registraron 60 expedientes de pacientes pediátricos a quienes se realizó orquiectomía y reporte de histopatología. Se encontraron 24 (40\%) casos con diagnóstico de torsión testicular: 19 (79\%) en el lado izquierdo y 5 (21\%) en el derecho. El ultrasonido doppler se efectuó en 19 (79\%) pacientes: 12 (63\%) reportaron ausencia de flujo vascular testicular y 7 (37\%) con flujo vascular (Cuadro 1).

Se reportaron 14 (23\%) casos con atrofia testicular: 9 (64\%) del lado derecho y 5 (36\%) del izquierdo. El diagnóstico preoperatorio más frecuente fue criptorquidia en 13 (93\%) y testículo ectópico en 1 (7\%) paciente. De éstos, 5 casos resultaron sugerentes de atrofia testicular, pero solo a $3(21 \%)$ se les realizó ultrasonido y a 2 se les colocó prótesis testicular (Cuadro 1).

Se detectaron 12 tumores testiculares (20\%), 6 de cada lado; los estudios de imagen se realizaron en $6(50 \%)$ pacientes: 5 con ultrasonido y 1 con ultrasonido y tomografía, todos con diagnóstico de tumor testicular. El reporte de patología fue: tumor germinal mixto en $9(75 \%)$ y germinal no seminomatoso en $3(35 \%)$ pacientes ( 2 del saco vitelino y 1 teratoma), ninguno con antecedente de criptorquidia (Cuadro 1).

Tres niños tuvieron testículo evanescente del lado izquierdo: 1 operado a los 9 meses y el resto 
a los 12 meses, ninguno contaba con estudios de imagen. El diagnóstico previo a la cirugía fue criptorquidia en dos casos y criptorquidia con atrofia testicular en el tercero. El acceso en $2(67 \%)$ pacientes fue por vía laparoscópica y en $1(33 \%)$ por vía inguinal. No se registraron complicaciones quirúrgicas (Cuadro 1).

Se encontró 1 (1\%) paciente con resto mülleriano y 6 con testículos normales, 5 de ellos con diagnóstico preoperatorio de atrofia testicular y 1 con torsión testicular (Cuadro 1).
En cuanto al grupo con torsión testicular, la edad promedio al momento de la intervención quirúrgica fue de 13 años (límites de 4 a 17 años) y la de pacientes con atrofia testicular de 8 años (límites de 1 a 16 años). En los 12 pacientes con tumores testiculares se registró un promedio de edad de 14 años (límites de 2 a 17 años) (Figura 1).

En los niños con torsión testicular, el tiempo transcurrido a partir del inicio de los síntomas hasta el momento de la intervención quirúrgica

Cuadro 1. Alteraciones testiculares tratadas con orquiectomía en pacientes pediátricos

\begin{tabular}{|l|c|c|c|c|c|}
\hline Causa & Número & Lado derecho & Lado izquierdo & Ultrasonido doppler & Prótesis testicular \\
\hline Torsión testicular & $24(40 \%)$ & 5 & 19 & 19 & 0 \\
\hline Atrofia testicular & $14(23.4 \%)$ & 9 & 5 & 3 & 2 \\
\hline Tumor testicular & $12(20 \%)$ & 6 & 6 & 7 & 0 \\
\hline Testículo evanescente & $3(5 \%)$ & 0 & 3 & 0 & 0 \\
\hline Resto mülleriano & $1(1.6 \%)$ & 1 & 0 & 0 & 0 \\
Testículo normal & $6(10 \%)$ & 1 & 5 & 0 & 1 \\
Total & $\mathbf{6 0}$ & $\mathbf{2 2}$ & $\mathbf{3 8}$ & $\mathbf{2 9}$ & $\mathbf{3}$
\end{tabular}

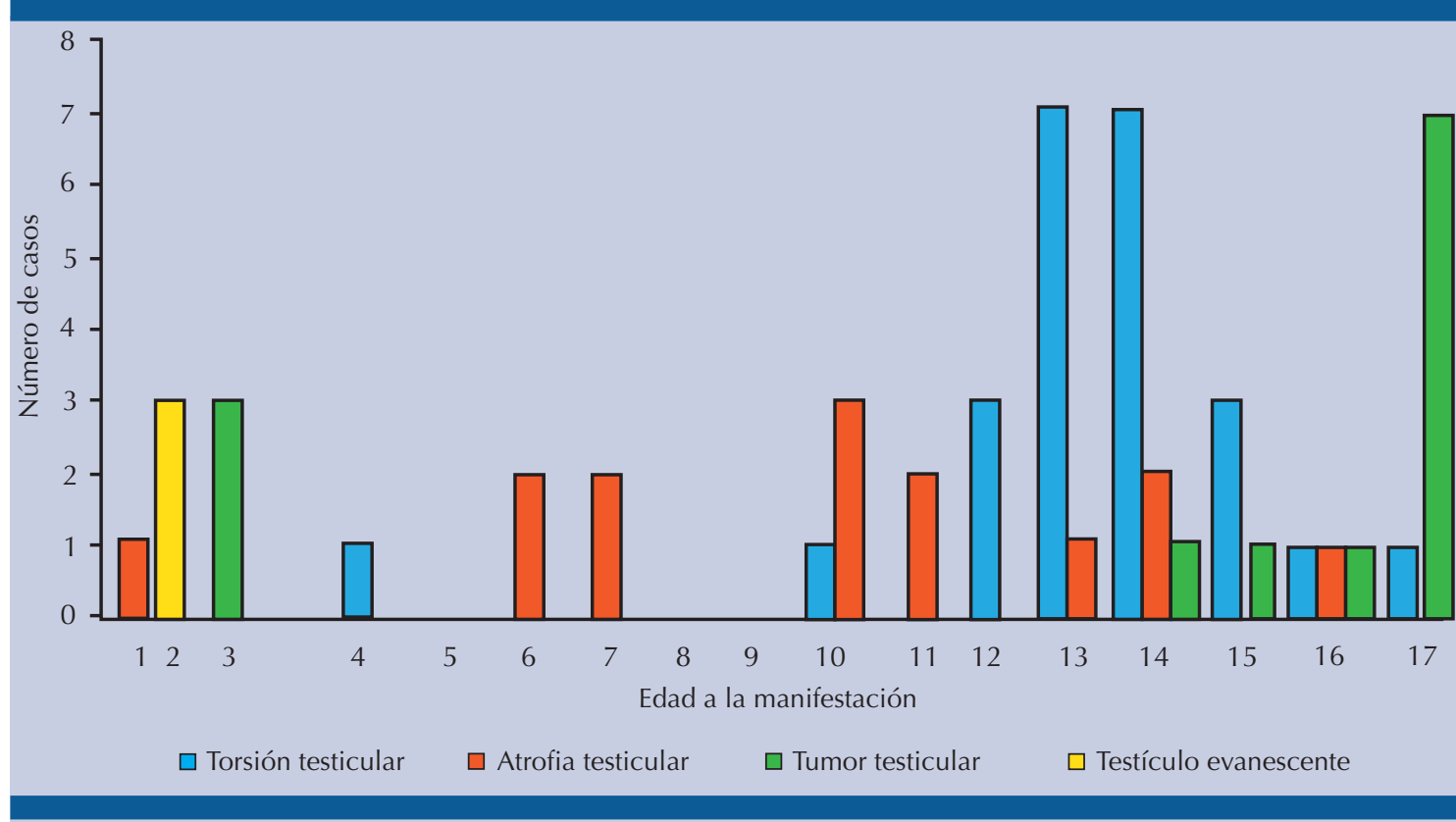

Figura 1. Alteraciones testiculares por edad. 
reportó un límite de 11 a 96 horas, con un promedio de $27.5 \mathrm{~h}$. Los pacientes se dividieron en tres grupos: 14 se intervinieron en un periodo de 12 a 24 horas, 9 de 24 a 48 horas y solo 1 en las primeras 28 horas (Figura 2).

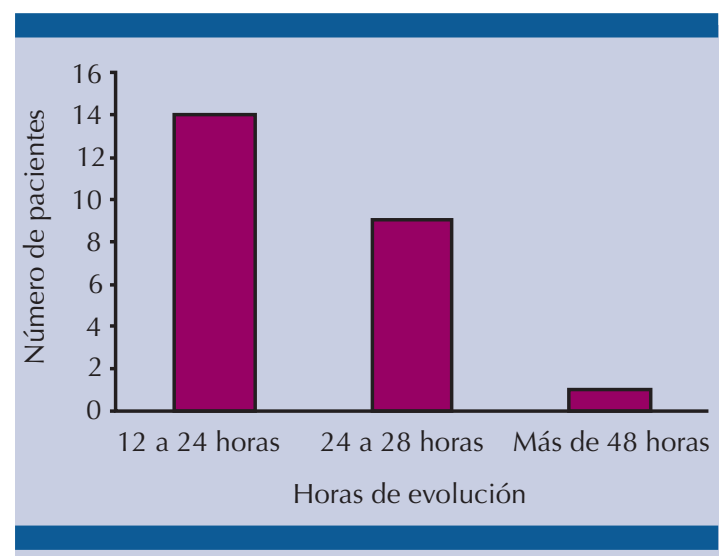

Figura 2. Tiempo de evolución de los pacientes con torsión testicular.

\section{DISCUSIÓN}

Al comparar nuestros resultados con los de otros autores encontramos que la alteración reportada fue similar; sin embargo, un estudio con 883 especímenes, llevado a cabo en Indiana (Estados Unidos), en un periodo de 20 años (1990 a 2009), con límites de edad de 0 a 20 años, reportó que el testículo evanescente fue la alteración más frecuente $(n=327 ; 37 \%)$, seguido de la atrofia por criptorquidia $(n=236$; $27 \%$ ) y la torsión testicular ( $n=125 ; 14 \%)$; los tumores testiculares correspondieron a 10\% (n =90) y se registraron 24 casos (3\%) con histología normal. ${ }^{13}$ La mayoría de los pacientes tuvo afectación del lado izquierdo y ocasionalmente se detectaron lesiones bilaterales. En 8 pacientes hubo hallazgos de restos suprarrenales. ${ }^{13}$

En nuestra serie el mayor número de casos correspondió a torsión $(43 \% ; n=24)$, seguido de atrofia $(23 \% ; n=14)$ y tumores testiculares $(20 \% ; n=12)$; el testículo evanescente se encontró en $5 \%(n=3)$ de los casos. Se reportaron
6 pacientes $(10 \%)$ con testículos sanos y 1 con resto mülleriano. El lado más afectado fue el izquierdo $(62 \%)$ y no se identificaron casos con afectación bilateral. La edad al momento de la manifestación de la torsión testicular fue entre los 4 y 17 años (promedio de 13 años), similar a lo reportado en la bibliografía. A 76\% se solicitó ultrasonido doppler, pero solo en $37 \%$ se detectó flujo vascular. No se registraron alteraciones en el periodo neonatal, comparado con otros estudios que refieren, incluso, $60 \% .^{1,13}$

El mayor número de casos de nuestra serie correspondió a torsión testicular, quizá debido a la demora en la intervención quirúrgica, que se llevó a cabo en las primeras 11 horas (en promedio a las 27 horas) de haber iniciado los síntomas y es probable que a los pacientes a quienes se les detectó flujo sanguíneo mediante ultrasonido doppler pudieron salvarse con una operación a tiempo.

Los pacientes con atrofia testicular registraron un promedio de edad de 9.2 años; el lado más afectado fue el derecho y el diagnóstico más común fue criptorquidia (70\%) y atrofia testicular (30\%). Solo en $15 \%$ se practicó el ultrasonido testicular, cuyo reporte más frecuente fue atrofia y disminución del flujo sanguíneo.

En cuanto a tumores testiculares primarios respecta, el rabdomiosarcoma es la neoplasia más común en pacientes de 15 y 16 años de edad, seguido de los tumores germinales (16 años). Por su parte, la leucemia linfoblástica representa el tumor secundario más frecuente y suele afectar el lado izquierdo del cuerpo. ${ }^{1,13}$ En nuestra serie, la mayoría de los pacientes tuvo tumor germinal mixto y el resto de subtipo germinal no seminomatoso, con edad promedio de 14 años (límites de 2 a 17 años). No se registró ningún caso con antecedente de criptorquidia.

En relación con la edad, los testículos evanescentes representan la alteración más frecuente reportada, cuyo diagnóstico se establece en los primeros 3 años de vida. ${ }^{13}$ En este estu- 
dio registramos 3 (5\%) casos con diagnóstico preoperatorio de criptorquidia, a quienes no se les practicaron estudios de imagen. El diagnóstico de testículo evanescente se estableció al momento de la intervención quirúrgica, en uno de ellos por abordaje laparoscópico.

Se reportaron 6 testículos normales (9.3\%), que en el diagnóstico preoperatorio 1 correspondió a torsión testicular y los otros 5 a atrofia testicular. Ningún paciente contó con ultrasonido. De los 60 casos incluidos en esta serie, solo a $3(5 \%)$ se les colocó una prótesis testicular.

\section{CONCLUSIONES}

El porcentaje de pacientes con alteraciones testiculares que requieren orquiectomía es elevado $(27 \%)$ en nuestro hospital. La principal causa obedece a torsión testicular, dato diferente a lo reportado por otros autores. El diagnóstico y tratamiento oportunos, establecidos mediante estudios ultrasonográficos, puede ayudar a disminuir este porcentaje.

\section{Conflicto de interés}

Los autores declaran no tener conflicto de interés.

\section{Financiamiento}

Los autores no recibieron ningún patrocinio para llevar a cabo esta investigación.

\section{REFERENCIAS}

1. Steinbrecher $\mathrm{H}$, Malone P. Testicular problems in children. J Pediatr Child Health 2008;18:264-267.

2. Srikumar BP, Gail B. Pediatric testicular problems. Pediatr Clin North Am 1998;45:813-830.

3. Marulaiah M, Gilhotra A, Moore L, et al. Testicular and paratesticular pathology in children: a 12-year histopathological review. World J Surg 2010;34:969-974.

4. Belman $\mathrm{A}$, Rushton $\mathrm{H}$. Is the vanished testis always a scrotal event? BJU Int 2001;87:480-483.

5. Grady W, Mitchell E, Carr C. Laparoscopic and histologic evaluation of the inguinal vanishing testis. J Urol 1998;52(5):866-869.

6. Herzog B, Hadziselimovic F, Strebel C. Primary and secondary testicular atrophy. Eur J Pediatr 1987;146(Suppl 2):S53-S55

7. Cost G, Bush C, Barber D, et al. Pediatric testicular torsion: demographics of national. orchiopexy versus orchiectomy rates. J Urol 2011;185:2459-2463.

8. Guía de práctica clínica. Abordaje diagnóstico del escroto agudo en el niño y el adolescente (Evidencias y Recomendaciones), México: Secretaria de Salud, 2010.

9. Hermann D. The pediatric acute scrotum. Pediatr Ann 1989;18(3):198-202, 204.

10. Baeza C, Martínez MI, Cortés R, et al. Orchiectomy for acute testicular torsión. Rev Mex Urol 2010;70(1):36-40.

11. Ringdahl E, Teague L. Testicular TOrsion. Am Fam Phys 2006;74(10):1739-1743.

12. Murphy F, Law H, Mushtaq I, et al. Testicular and paratesticular pathology in infants and children:the histopathological experience of a tertiary paediatric unitover a 17 year period. Pediatr Surg Int 2007;23:867-872.

13. Fan $R$, Zhang J, Cheng $L$, et al. Testicular and paratesticular pathology in the pediatric population: A 20 year experience at Riley hospital for children. Pathol Res Pract 2013;209:404-408. 